\title{
Phase-Matched Third-Harmonic Generation of Nd: Glass-Laser Picosecond Pulses in a New Cyanine-Dye Solution
}

\author{
C. Schwan ${ }^{1}$, A. Penzkofer ${ }^{1}$, N. J. Marx $^{2}$, K. H. Drexhage ${ }^{2}$ \\ 1 Naturwissenschaftliche Fakultät II-Physik, Universität Regensburg, D-93040 Regensburg, Germany (Fax: +49-941/943-2754, \\ Tel.: +49-941/943-2107) \\ 2 Universität-Gesamthochschule Siegen, D-57076 Siegen, Germany (Fax: +49-271/740-2330, Tel.: +49-271/740-4187)
}

Received 27 April 1993/Accepted 2 June 1993

\begin{abstract}
The phase-matched direct tripling of picosecond light pulses of a mode-locked Nd:glass laser in a new cyanine dye PMC is studied. The solvents trifluoroethanol (TFE) and hexafluoroisopropanol (HFIP) are applied. The $S_{0}-S_{1}$ absorption peak of the dye is around $\lambda=480 \mathrm{~nm}$ and the absorption cross section at the third-harmonic wavelength of $\lambda_{3}=351.3 \mathrm{~nm}$ is only $\sigma_{3} \approx 1 \times 10^{-19} \mathrm{~cm}^{2}$. Phase-matching occurred at concentrations of $\mathrm{C}_{\mathrm{PM}}=0.0874 \mathrm{~mol} / \mathrm{dm}^{3}$ in HFIP and $0.1088 \mathrm{~mol} / \mathrm{dm}^{3}$ in TFE. A third-harmonic energy conversion efficiency of $\eta_{\mathrm{E}} \approx 0.01$ was achieved at a pump-laser peak intensity of $I_{0 \mathrm{~L}} \approx 2.5 \times 10^{11} \mathrm{~W} / \mathrm{cm}^{2}$ in a $5 \mathrm{~mm}$ long sample of PMC in TFE. The conversion efficiency is limited by destruction of phase-matching due to the intensity-dependent nonlinear refractive index of the dye solutions.
\end{abstract}

PACS: 42.65

Efficient frequency tripling of laser radiation is performed generally in a two-step process first generating the secondharmonic light in a phase-matched nonlinear optical crystal and then frequency mixing the fundamental and the secondharmonic light in another phase-matched nonlinear optical crystal [1-3]. The second-order nonlinear optical susceptibility $\chi^{(2)}$ is responsible for these conversion processes. Direct (single-step) angle-tuned phase-matched third-harmonic generation of $\mathrm{Nd}$ :laser radiation was realized in the crystals $\mathrm{LiIO}_{3}$ [4], $\mathrm{CaCO}_{3}$ [5], and $\beta-\mathrm{BaB}_{2} \mathrm{O}_{4}$ [6]. In the vapor phase efficient phase-matched third-harmonic generation of $\mathrm{Nd}$ :laser radiation was achieved in mixtures of alkali vapors and noble gases [2,7-13]. The direct third-harmonic generation is caused by the third-order nonlinear optical susceptibility $\chi^{(3)}$.

Phase-matched third-harmonic generation of Nd: laser radiation in organic dye solutions was studied in [14-19]. Dyes having the $S_{0}-S_{1}$ absorption peak between the fundamental and third-harmonic frequency were selected for a low absorption cross section at the third-harmonic frequency. They were dissolved in a solvent of low normal refractive index dispersion. Phase-matching was achieved at a certain dye concentration at which the anomalous refractive index dispersion of the dyes compensated the normal refractive index dispersion of the solvents. For the dye PYC dissolved in hexafluoroisopropanol the absorption cross section at the third-harmonic frequency $\nu_{3}$ was $\sigma_{3}=3.55 \times 10^{-18} \mathrm{~cm}^{2}$, the two-photon absorption cross section was $\sigma_{\mathrm{LL}}^{(2)}=1.8 \times 10^{-49} \mathrm{~cm}^{4} \mathrm{~s}$ and the excitedstate absorption cross section of third-harmonic light was $\sigma_{3, \mathrm{ex}}=2.6 \times 10^{-16} \mathrm{~cm}^{2}$ [18]. A maximum third-harmonic energy conversion efficiency of $\eta_{\mathrm{E}}=2 \times 10^{-4}$ was achieved for input peak intensities $I_{0 \mathrm{~L}}>10^{11} \mathrm{~W} / \mathrm{cm}^{2}$ (sample thickness $l=0.2 \mathrm{~mm}$ ) [18]. The maximum obtainable conversion efficiency was limited by i) the small interaction length because of residual dye absorption at the third-harmonic frequency and ii) by two-photon dye absorption at twice the fundamental laser frequency and concomitant excited-state absorption of the dye.

In this paper third-harmonic generation in a new dye with very low absorption cross section at the third-harmonic frequency and negligible absorption at twice the fundamental laser frequency is investigated. A third-harmonic energy conversion efficiency of $\eta_{\mathrm{E}} \approx 1 \%$ is obtained at a fundamental peak pulse intensity of $I_{0 \mathrm{~L}} \simeq 2.5 \times 10^{11} \mathrm{~W} / \mathrm{cm}^{2}$ for the new dye PMC in 2,2,2-trifluoroethanol (TFE). The conversion efficiency is limited by phase-mismatching due to intensity-dependent refractive index changes [19-21].

\section{Dye and Solvent Characterization}

We tried to find a dye with very low absorption at the thirdharmonic wavelength $\lambda_{3}=351.3 \mathrm{~nm}$ of our Nd:phosphate glass laser and low absorption of the second-harmonic wavelength $\lambda_{3}=527 \mathrm{~nm}$ (avoiding of two-photon absorption and concomitant excited-state absorption). Screening a large number of compounds out of our lab collection we found a correlation between the molecular structure and the absorption minimum on the short-wavelength side of the $S_{0}-S_{1}$ transition. In general, the absorption cross section at this minimum turned out to be particularly low, if the $\pi$-electron system responsible for light absorption was simple and un- 


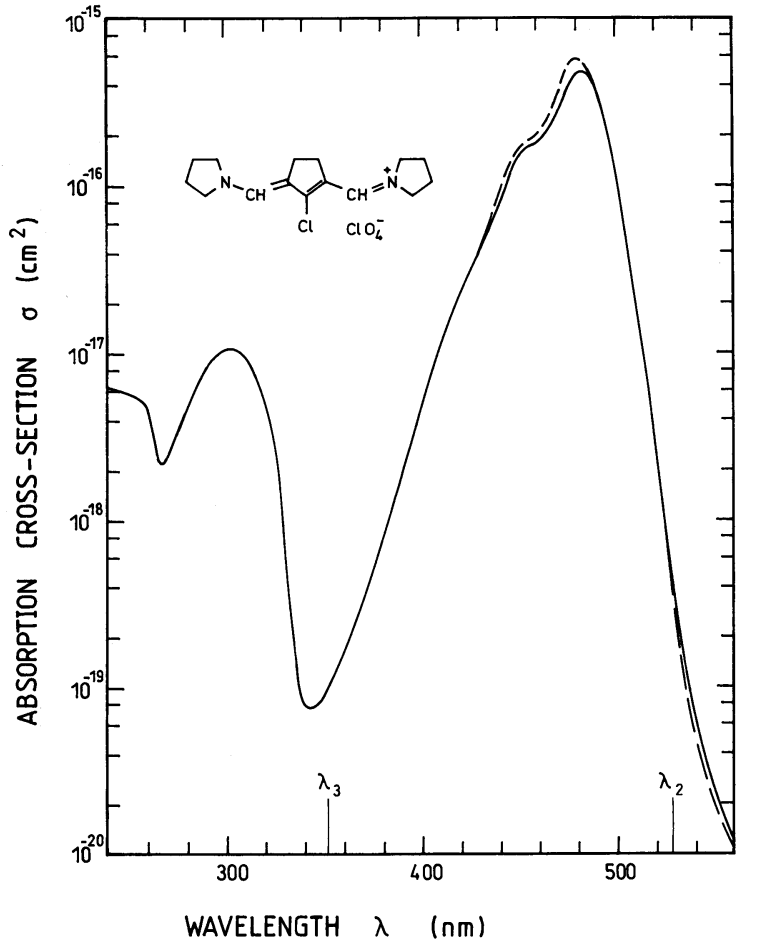

Fig. 1. Absorption cross-section spectra of dye PMC in 2,2,2-trifluoroethanol (TFE, solid curve) and 1,1,1,3,3,3-hexafluoro-2-propanol (HFIP, dashed curve). Structural formula of PMC is inserted

branched. With this in mind we then proceeded to synthesize several new cyanine dyes. One of them, a pentamethine cyanine dye (PMC), seemed to be most promising for thirdharmonic generation, as it showed an extremely low absorption cross section at wavelength $\lambda_{3}$ and low absorption at the second-harmonic wavelength $\lambda_{2}$. The structural formula of the new dye PMC is shown in Fig. 1 together with the absorption cross section spectra in the solvents 2,2,2trifluoroethanol (TFE) and 1,1,1,3,3,3-hexafluoro-2-propanol (HFIP).

The dye PMC was synthesized in analogy to [22]. The non-optimized conditions are as follows. A solution of $42 \mathrm{~g}(0.42 \mathrm{~mol})$ of $\mathrm{N}$-formylpyrrolidine in $700 \mathrm{ml}$ of dry chloroform was chilled in an ice bath and $45 \mathrm{ml}(0.48 \mathrm{~mol})$ of phosphorus oxychloride in $200 \mathrm{ml}$ of chloroform was added dropwise with vigorous stirring. Stirring was continued for $2 \mathrm{~h}$ while the temperature was allowed to rise to $20^{\circ} \mathrm{C}$. After cooling to $5^{\circ} \mathrm{C}, 35 \mathrm{~g}(0.42 \mathrm{~mol})$ of cyclopentanone in $200 \mathrm{ml}$ of chloroform was added dropwise with stirring. The solution was refluxed for $3 \mathrm{~h}$. After cooling and vacuumevaporation of the solvents the dark-red oily residue was dissolved in 21 of water. On addition of $10 \mathrm{~g}$ of sodium perchlorate, dissolved in water, the pentamethine cyanine perchlorate (PMC) precipitated. It was collected by filtration, washed successively with small amounts of water, ethanol and diethyl ether to yield $20 \mathrm{~g}$ of crude PMC. The product was recrystallized three times from acetonitrile and was obtained as large violet needles.

The absorption cross section spectra of the dye in the solvents TFE and HFIP are shown in Fig. 1. The $S_{0^{-}}$ $S_{1}$ absorption peak is at $\lambda_{\mathrm{p}}=484 \mathrm{~nm}$ for TFE $\left(\sigma_{\mathrm{p}}=\right.$ $\left.4.9 \times 10^{-16} \mathrm{~cm}^{2}\right)$ and at $\lambda_{\mathrm{p}}=481 \mathrm{~nm}$ for $\operatorname{HFIP}\left(\sigma_{\mathrm{p}}=\right.$
$5.8 \times 10^{-16} \mathrm{~cm}^{2}$ ). A deep absorption minimum between the first absorption maximum and further short-wavelength singlet absorption maxima is located at $342 \mathrm{~nm}\left(\sigma_{\mathrm{m}}=\right.$ $7.5 \times 10^{-20} \mathrm{~cm}^{2}$ for both solvents). At the third-harmonic wavelength $\lambda_{3}=351.3 \mathrm{~nm}$ of our $\mathrm{Nd}$ :phosphate glass laser (wavelength $\lambda_{\mathrm{L}}=1.054 \mu \mathrm{m}$ ) the absorption cross section is $\sigma_{3} \approx 1 \times 10^{-19} \mathrm{~cm}^{2}$. The absorption cross section at the second-harmonic wavelength $\lambda_{2}=527 \mathrm{~nm}$ is $\sigma_{2} \approx 4.5 \times 10^{-19} \mathrm{~cm}^{2}$. The $S_{0}-S_{1}$ transition is offresonant to the second-harmonic wavelength and therefore two-photon absorption does not influence third-harmonic generation $[19,23,24]$. The anomalous dispersion of the refractive index of the dye in the $S_{1}$-absorption band allows phase-matching at a certain dye concentration.

The solubility of PMC in the solvents methanol, TFE, and HFIP is $0.01 \mathrm{~mol} / \mathrm{dm}^{3}, 0.59 \mathrm{~mol} / \mathrm{dm}^{3}$, and $2 \mathrm{~mol} / \mathrm{dm}^{3}$, respectively. The comparatively low solubility of PMC in methanol does not allow phase-matched third-harmonic generation in this solvent.

The stability of the dye is high in TFE and low in HFIP. The temporal reduction of the absorption coefficient $\alpha_{\mathrm{p}}$ at the $S_{0}-S_{1}$ absorption peak due to heat treatment and light exposure is shown in Fig. 2a,b for the dye in HFIP and TFE, respectively, where the ratio $\alpha_{\mathrm{p}}(t) / \alpha_{\mathrm{p}}(0)=\ln T_{\mathrm{p}}(t) / \ln T_{\mathrm{p}}(0)$ is plotted versus time $\left(T_{\mathrm{p}}(t)\right.$ is the transmission at wavelength $\lambda_{\mathrm{p}}$ and time $t$ ).

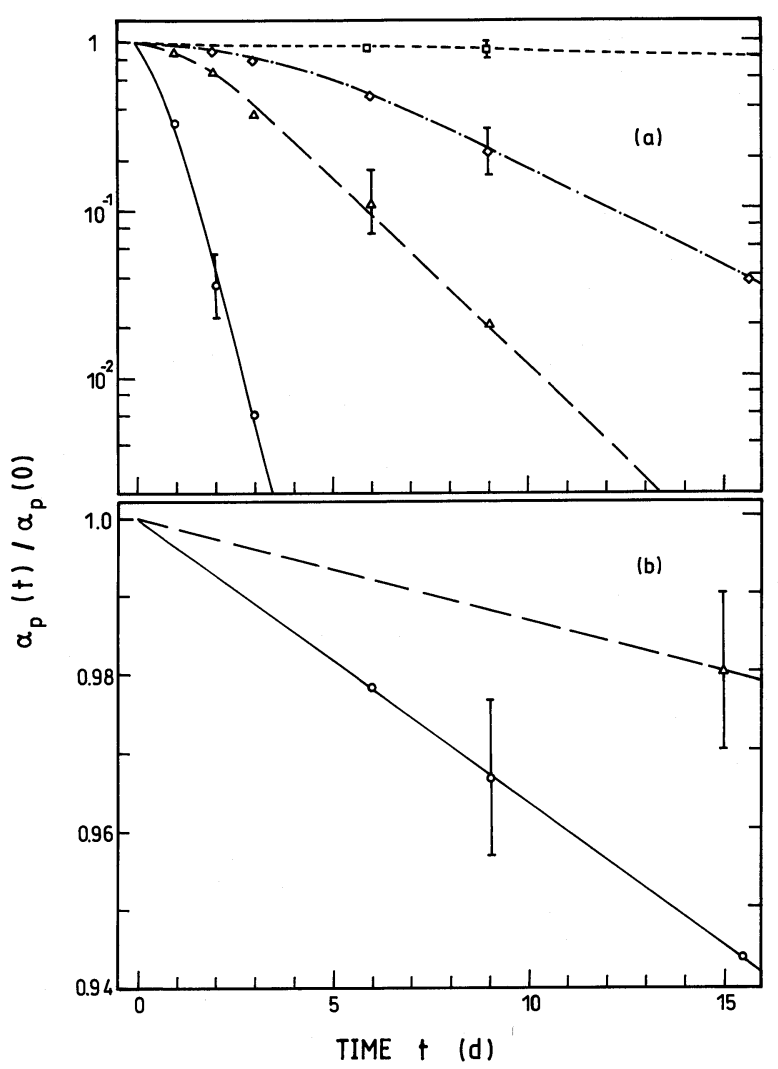

Fig. 2a, b. Normalized peak $S_{0}-S_{1}$ absorption coefficient $\alpha_{\mathrm{p}}(t) / \alpha_{\mathrm{p}}(0)$ vs time $t$ in days for dye PMC in HFIP (a) and in TFE (b). Circles and solid curves, dye solution an $40^{\circ} \mathrm{C}$ in the dark. Diamonds and dashdotted curve, $20^{\circ} \mathrm{C}$ in the dark. Squares and short-dashed curve, $4^{\circ} \mathrm{C}$ in the dark. Triangles and long-dashed curves, $20^{\circ} \mathrm{C}$ under daylight in a room 


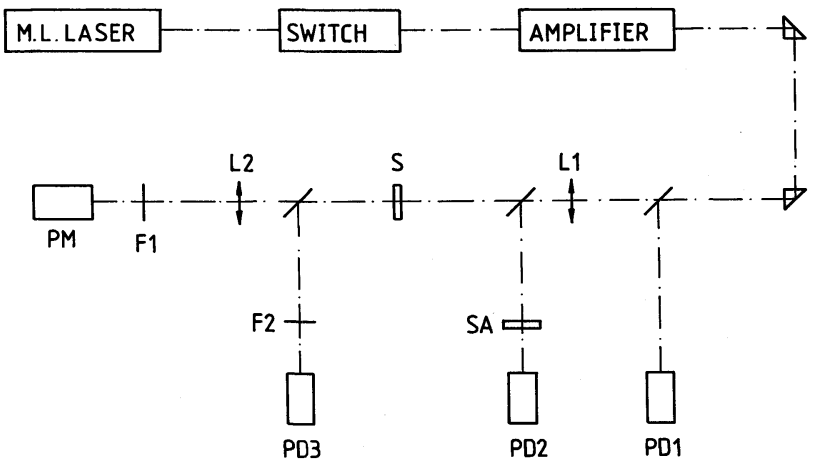

Fig. 3. Experimental arrangement. L1, L2, lenses; S, dye sample; SA, saturable absorber cell. F1, F2, filters; PD1-PD3, photodetectors; PM, photomultiplier

\section{Experimental}

The experimental setup for the measurement of the thirdharmonic conversion efficiency and of the fundamental laser pulse transmission is shown in Fig. 3. An active [25] (acousto-optic mode-locker, IntraAction model ML-50Q) and passive [26] (saturable absorber Kodak dye No. 9860) mode-locked $\mathrm{Nd}$ :phosphate glass laser (laser glass, Hoya LHG7) is applied for the picosecond pulse generation (wavelength $\lambda_{\mathrm{L}}=1054 \mathrm{~nm}$, pulse duration $\Delta t_{\mathrm{L}} \approx 5 \mathrm{ps}$ ). A single pulse is selected by a Kerr-cell shutter [27] and the selected pulse is amplified in a $\mathrm{Nd}$ :phosphate glass-laser amplifier (glass, Hoya LHG5, amplified pulse energy up to $10 \mathrm{~mJ}$ ).

The pump pulse intensity is varied by changing the voltage of the power supply of the laser amplifier and by using focusing lenses L1. The peak intensity of the pumplaser pulses is determined by energy-transmission detection through a saturable dye SA [28] (Kođak dye No. 9860, photodetectors PD1 and PD2). The laser-pulse intensity was increased up to the surface damage of the fused-silica cells $\left(I_{0 \mathrm{~L}} \approx 8 \times 10^{11} \mathrm{~W} / \mathrm{cm}^{2}\right)[20,29-32]$. The generated third-harmonic light is detected by the photomultiplier PM (Valvo type TUVP56, S20 spectral characteristic). The thirdharmonic energy-conversion efficiency $\eta_{\mathrm{E}}$ is determined by relating the signal of the photomultiplier PM to the signal of the photodetector PD1. The photomultiplier PM and the photodetector PD1 are calibrated by a pyroelectric joulemeter (Molectron, type J3-09). The pump-pulse transmission through the dye solutions and through the solvents was measured by the photodetectors PD3 and PD1.

\section{Experimental Results}

The experimental third-harmonic energy conversion efficiency $\eta_{\mathrm{E}}$ versus dye concentration $C$ is displayed in Fig. 4 . The circles of part (a) belong to a $1 \mathrm{~mm}$ sample of PMC in HFIP. The phase-matching concentration is $C_{\mathrm{PM}}=0.0874 \pm$ $0.0005 \mathrm{~mol} \mathrm{dm}^{-3}$. The circles in part (b) belong to a $1 \mathrm{~mm}$ sample of PMC in TFE. Here, the phase-matching concentration is $C_{\mathrm{PM}}=0.1088 \pm 0.0005 \mathrm{~mol} \mathrm{dm}^{-3}$. In both cases the pump laser intensity is $I_{0 \mathrm{~L}}=9.2 \times 10^{9} \mathrm{~W} / \mathrm{cm}^{2}$. The dots in part (b) belong to a $5 \mathrm{~mm}$ sample of PMC in TFE at a pump laser intensity of $I_{0 \mathrm{~L}} \approx 2.5 \times 10^{11} \mathrm{~W} / \mathrm{cm}^{2}$. At this high

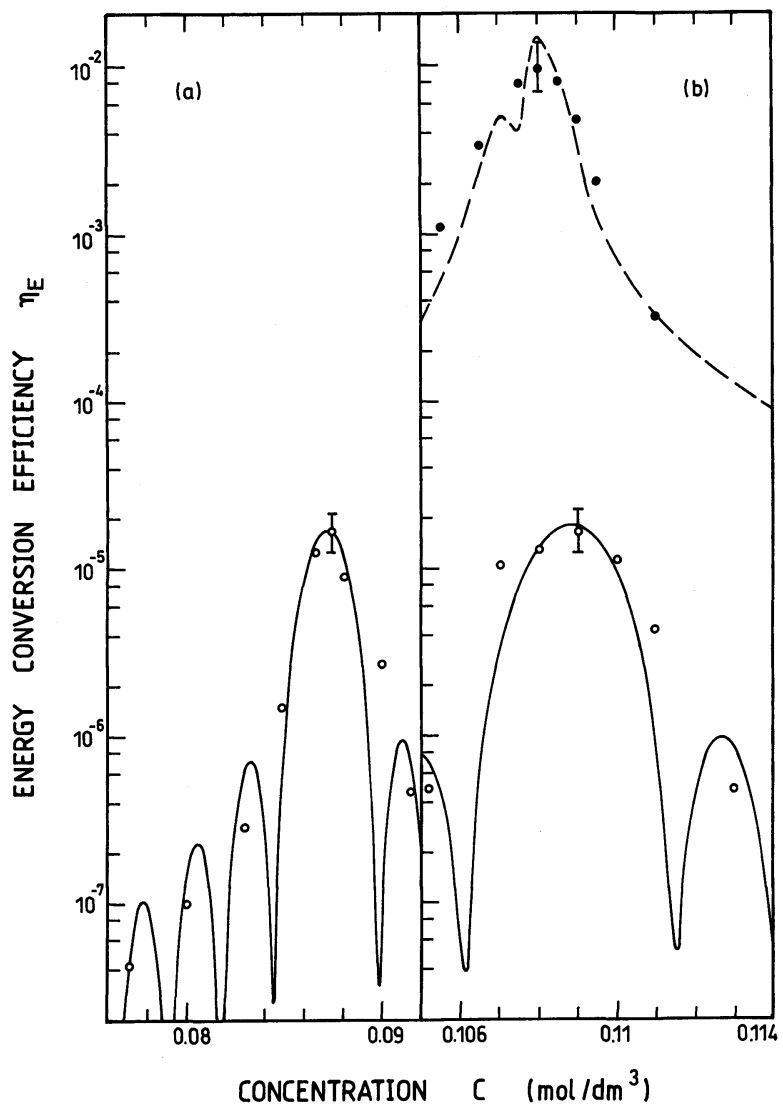

Fig. 4a,b. Third-harmonic energy-conversion efficiency vs dye concentration. Circles and solid curves, pump-laser peak intensity $I_{0 \mathrm{~L}}=$ $9.2 \times 10^{9} \mathrm{~W} / \mathrm{cm}^{2}$. Dots and dashed curve, $I_{0 \mathrm{~L}}=2.5 \times 10^{11} \mathrm{~W} / \mathrm{cm}^{2}$ a Dye PMC in HFIP. b Dye PMC in TFE. The curves are calculated with the data of Table 1

pump intensity the optimum dye concentration is shifted to a slightly lower value of $C_{\mathrm{opt}}=0.1080 \pm 0.0005 \mathrm{~mol} \mathrm{dm}^{-3}$. The shift of the optimum concentration is thought to be caused by the dependence of the refractive indices on the laser intensity (see below). The maximum energy conversion efficiency obtained is $\eta_{\mathrm{E}}=0.01 \pm 0.003$.

The third-harmonic energy-conversion efficiency versus pump-laser peak intensity in $5 \mathrm{~mm}$ dye samples is shown in Fig. 5 for the solvent TFE and in Fig. 6 for the solvent HFIP. The circles in both figures apply to low-intensity phase-matched dye solutions. The triangles in Fig. 5 belong to a dye concentration of $C=0.1109 \mathrm{~mol} / \mathrm{dm}^{3}$. At high pump-pulse intensities, the conversion efficiency obtained under low-intensity phase-matched conditions levels off and exhibits a maximum at $I_{0 \mathrm{~L}} \approx 3 \times 10^{11} \mathrm{~W} / \mathrm{cm}^{2}$. Under nonphase-matched conditions (triangles of Fig. 5) the quadratic dependence of $\eta_{\mathrm{E}}$ versus $I_{0 \mathrm{~L}}$ remains approximately valid up to the highest applied pump-pulse intensities.

The energy transmission $T_{\mathrm{E}}$ of the fundamental laser pulses through the solvents and the phase-matched dye solutions is shown in Fig. 7. The sample length is $4 \mathrm{~cm}$. From the low-intensity transmissions the absorption coefficients of the solvents and the absorption cross section of the dye at the laser frequency are derived (see Table 1). Above $8 \times 10^{10} \mathrm{~W} / \mathrm{cm}^{2}$ in the case of HFIP and above $5 \times 10^{10} \mathrm{~W} / \mathrm{cm}^{2}$ in the case of TFE the transmission drops 


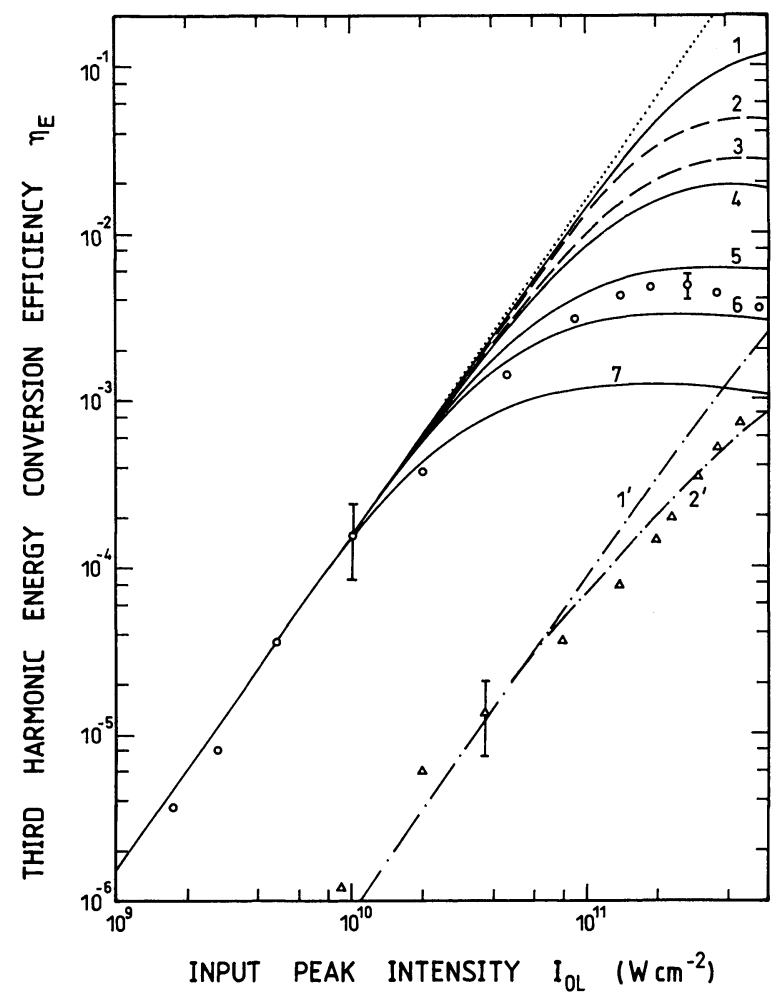

Fig. 5. Third-harmonic energy-conversion efficiency vs pump-laser input peak intensity for dye PMC in TFE. Circles and curves 1-7, lowintensity phase-matched dye concentration $C_{\mathrm{PM}}=0.1088 \mathrm{~mol} / \mathrm{dm}^{3}$. Triangles and dash-dotted curves $1^{\prime}$ and $2^{\prime}, C=0.1109 \mathrm{~mol} / \mathrm{dm}^{3}$. The curves are calculated for $\left(1,1^{\prime}\right)$ without nonlinear refractive-index contribution, (2) $\kappa=1.5 \times 10^{-44}$, (3) 0 , (4) $6 \times 10^{-44}$, (5) $9 \times 10^{-44}$, (6) $1.2 \times 10^{-43}$, (7) $1.8 \times 10^{-43},\left(2^{\prime}\right) 9.6 \times 10^{-44} \mathrm{Cm}^{4} \mathrm{~V}^{-3} \mathrm{~s}^{-1}$. Curve 1 also belongs to $\kappa=3 \times 10^{-44} \mathrm{Cm}^{4} \mathrm{~V}^{-3} \mathrm{~s}^{-1}$. The dotted curve belongs to (19) with $\Delta k=0$ and $C_{\mathrm{PM}}=0.1088 \mathrm{~mol} / \mathrm{dm}^{3}$. Other parameters are taken from Table 1

rapidly. The onset of transmission reduction scales up to $I_{0 \mathrm{~L}} \approx 6 \times 10^{11} \mathrm{~W} / \mathrm{cm}^{2}$ (HFIP) and $4 \times 10^{11} \mathrm{~W} / \mathrm{cm}^{2}$ (TFE) for samples of $5 \mathrm{~mm}$ length. This behaviour was verified by transmission measurements in $5 \mathrm{~mm}$ samples. The decrease of transmission is mainly due to stimulated Raman scattering [33] (see below). White-light generation was observed for $I_{0 \mathrm{~L}} \gtrsim 2 \times 10^{11} \mathrm{~W} / \mathrm{cm}^{2}$ in the $4 \mathrm{~cm}$ long cells filled with either the solvents or the dye solutions $[34,35]$.

Fig. 7. Energy transmission of pump-laser pulses through solvents and dye solutions. Sample length $l=4 \mathrm{~cm}$. Open circles and solid curve 1 , HFIP. Open triangles and solid curve 2, 0.0874 molar PMC in HFIP. Filled circles and dashed curve 1', TFE. Filled triangles and dashed curve $2^{\prime}, 0.1088$ molar PMC in TFE

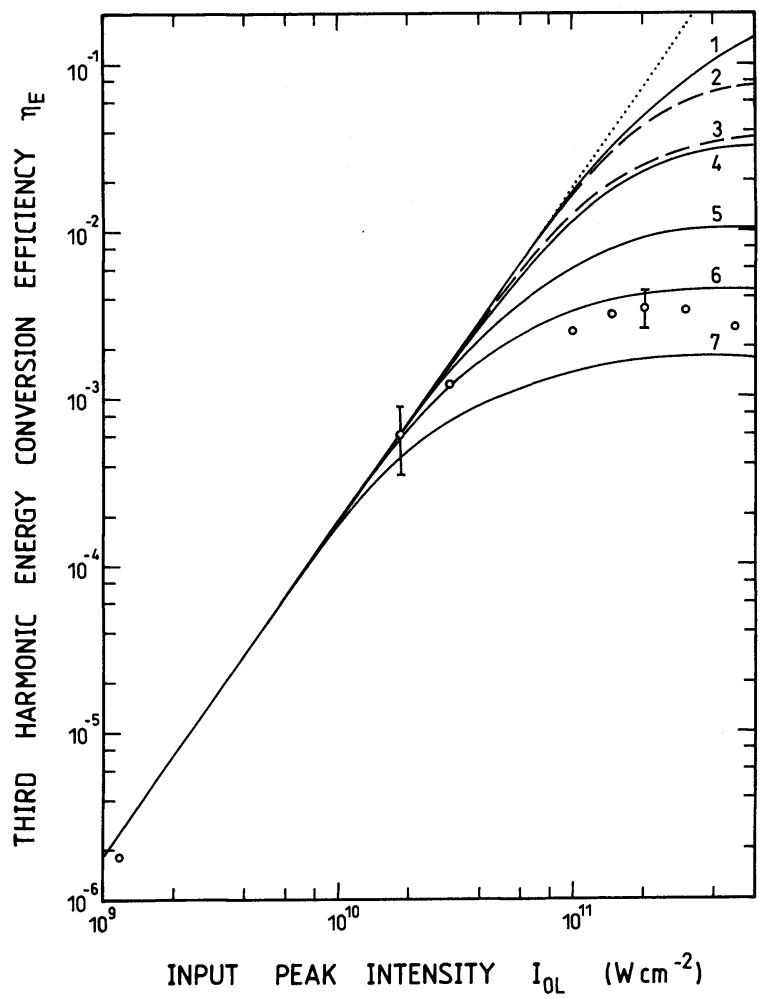

Fig. 6. Third-harmonic energy-conversion efficiency $\eta_{\mathrm{E}}$ vs pumppulse input peak intensity $I_{0 \mathrm{~L}}$ for 0.0874 molar PMC in HFIP (lowintensity phase-matched). Circles are experimental data. Curves are calculated for (1) without nonlinear refractive-index contribution, (2) $\kappa=1.5 \times 10^{-44}$, (3) 0 , (4) $6 \times 10^{-44}$, (5) $9 \times 10^{-44}$, (6) $1.2 \times 10^{-43}$, (7) $1.8 \times 10^{-43} \mathrm{Cm}^{4} \mathrm{~V}^{-3} \mathrm{~s}^{-1}$. Curve 1 also applies to $\kappa=3 \times$ $10^{-44} \mathrm{Cm}^{4} \mathrm{~V}^{-3} \mathrm{~s}^{-1}$. Dotted curve belongs to (19) with $\Delta k=0$. Other parameters are taken from Table 1

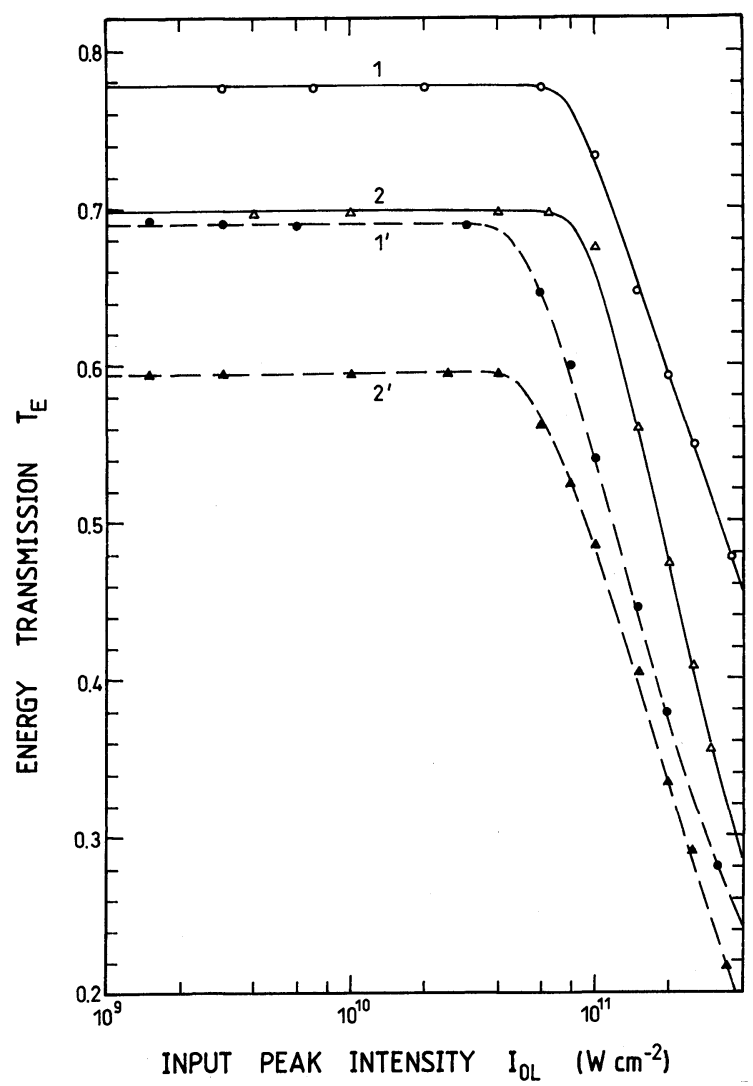


Table 1. Parameters of solvents TFE (2,2,2-trifluoroethanol) and HFIP (1,1,1,3,3,3-hexafluoro-2-propanol), of phase-matched solutions of dye PMC in TFE and HFIP, and of solute dye PMC. Wavelengths $\lambda_{\mathrm{L}}=1054 \mathrm{~nm}, \lambda_{3}=351.3 \mathrm{~nm}$. Conversion factors between SI units (used here) and esu units are $\gamma^{(3)}(\mathrm{esu})=8.0888 \times 10^{24} \gamma^{(3)}(\mathrm{SI})$ and $\chi^{(3)}(\mathrm{esu})=\left(9 \times 10^{8} / 4 \pi\right) \chi^{(3)}(\mathrm{SI})[49]$

\begin{tabular}{|c|c|c|c|}
\hline Parameter & TFE & HFIP & References \\
\hline$\alpha_{\mathrm{L}, \mathrm{S}}\left[\mathrm{cm}^{-1}\right]$ & 0.09 & 0.063 & Fig. 7 \\
\hline$n_{\mathrm{L}, \mathrm{S}}$ & 1.2863 & 1.2718 & {$[36]$} \\
\hline$n_{3, \mathrm{~s}}$ & 1.3005 & 1.2832 & {$[36]$} \\
\hline$\chi_{\mathrm{THG}, \mathrm{S}}^{(3)}\left[\mathrm{m}^{2} \mathrm{~V}^{-2}\right]$ & $2.4 \times 10^{-23}$ & $1.4 \times 10^{-23}$ & [18] (HFIP); this work \\
\hline$n_{2, \mathrm{LL}, \mathrm{S}}\left[\mathrm{m}^{2} \mathrm{~V}^{-2}\right]$ & $(1.2 \pm 0.2) 10^{-22}$ & $(1 \pm 0.2) 10^{-22}$ & This work \\
\hline$n_{2, \mathrm{~L} 3, \mathrm{~S}}\left[\mathrm{~m}^{2} \mathrm{~V}^{-2}\right]$ & $2.4 \times 10^{-22}$ & $2 \times 10^{-22}$ & $n_{2, \mathrm{~L} 3, \mathrm{~S}} \approx 2 n_{2, \mathrm{LL}, \mathrm{S}}$ \\
\hline$n_{2,33, \mathrm{~s}}\left[\mathrm{~m}^{2} \mathrm{~V}^{-2}\right]$ & $1.2 \times 10^{-22}$ & $1 \times 10^{-22}$ & $n_{2,33, \mathrm{~S}} \approx 2 n_{2, \mathrm{LL}, \mathrm{S}}$ \\
\hline \multicolumn{4}{|l|}{ Phase-matched solutions } \\
\hline$C_{\mathrm{PM}}\left[\mathrm{mol} \mathrm{dm}^{-3}\right]$ & 0.1088 & 0.0874 & Fig. 4 \\
\hline$\alpha_{\mathrm{L}}\left[\mathrm{cm}^{-1}\right]$ & 0.13 & 0.093 & Fig. 7 \\
\hline$n_{2, \mathrm{LL}}\left[\mathrm{m}^{2} \mathrm{~V}^{-2}\right]$ & $2.3 \times 10^{-22}$ & $2.2 \times 10^{-22}$ & $(14-18)$ \\
\hline$n_{2, \mathrm{~L} 3}\left[\mathrm{~m}^{2} \mathrm{~V}^{-2}\right]$ & $-7.9 \times 10^{-23}$ & $-1.4 \times 10^{-22}$ & $(14-18)$ \\
\hline$n_{2,33}\left[\mathrm{~m}^{2} \mathrm{~V}^{-2}\right]$ & $3.5 \times 10^{-22}$ & $3.5 \times 10^{-22}$ & $(14-18)$ \\
\hline \multicolumn{4}{|l|}{ Solute (dye PMC) } \\
\hline$\sigma_{\mathrm{L}}\left[\mathrm{cm}^{2}\right]$ & $4.7 \times 10^{-24}$ & $5.1 \times 10^{-24}$ & Fig. 6 \\
\hline$\sigma_{3}\left[\mathrm{~cm}^{2}\right]$ & $1 \times 10^{-19}$ & $1 \times 10^{-19}$ & Fig. 1 \\
\hline$\gamma_{\mathrm{THG}, \mathrm{D}}^{(3)}\left[\mathrm{Cm}^{4} \mathrm{~V}^{-3}\right]$ & $-3.3 \times 10^{-60}$ & $-3.3 \times 10^{-60}$ & This work \\
\hline$\gamma_{\mathrm{D}}^{(1)^{\prime}}\left(-\omega_{\mathrm{L}}, \omega_{\mathrm{L}}\right)\left[\mathrm{Cm}^{2} \mathrm{~V}^{-1}\right]$ & $4.075 \times 10^{-39}$ & $4.075 \times 10^{-39}$ & (6) and [36] \\
\hline$\gamma_{\mathbf{D}}^{(1)^{\prime}}\left(-\omega_{3}, \omega_{3}\right)\left[\mathrm{Cm}^{2} \mathrm{~V}^{-1}\right]$ & 0 & 0 & (6) and [36] \\
\hline$\gamma_{\mathrm{D}}^{(3)^{\prime}}\left(-\omega_{\mathrm{L}} ; \omega_{\mathrm{L}},-\omega_{\mathrm{L}}, \omega_{\mathrm{L}}\right)\left[\mathrm{Cm}^{4} \mathrm{~V}^{-3}\right]$ & $2.9 \times 10^{-60}$ & $3.8 \times 10^{-60}$ & $(16)$ \\
\hline$\gamma_{\mathrm{D}}^{(3)^{\prime}}\left(-\omega_{\mathrm{L}} ; \omega_{3},-\omega_{3}, \omega_{\mathrm{L}}\right)\left[\mathrm{Cm}^{4} \mathrm{~V}^{-3}\right]$ & $-4.1 \times 10^{-60}$ & $-5.6 \times 10^{-60}$ & (17) \\
\hline
\end{tabular}

\section{Discussion}

First, a general equation system is presented for thirdharmonic generation, then the third-harmonic nonlinear susceptibilities are determined, and finally the limitations of the third-harmonic conversion efficiency at high pump-laser intensities are analyzed.

\subsection{General Equation System}

A theoretical analysis of third-harmonic generation in dye solutions was given in [19]. Here, the equation system of light-mater interaction simplifies considerably since twophoton absorption (no resonant level at $2 \nu_{\mathrm{L}}$ ) and excitedstate absorption (weak absorption at $3 \nu_{\mathrm{L}}$ ) are negligible. The pump-pulse propagation and the third-harmonic generation along the propagation direction $z$ are described by (1) and (2) for the electrical field amplitudes of the pump light $E_{\mathrm{L} 0}$ and the third-harmonic light $\mathrm{E}_{30}$ :

$$
\begin{aligned}
\frac{\partial E_{\mathrm{L} 0}}{\partial z}= & -\frac{\alpha_{\mathrm{L}}}{2} E_{\mathrm{L} 0}-\frac{\mathrm{i} \omega_{\mathrm{L}}}{2 n_{\mathrm{L}} c_{0}}\left[3 \chi_{\mathrm{THG}}^{(3) *} E_{\mathrm{L} 0}^{* 2} E_{30} \exp (-\mathrm{i} \Delta k z)\right. \\
& \left.+n_{\mathrm{L}} n_{2, \mathrm{~L} 3}\left|E_{30}\right|^{2} E_{\mathrm{L} 0}+n_{\mathrm{L}} n_{2, \mathrm{LL}}\left|E_{\mathrm{L} 0}\right|^{2} E_{\mathrm{L} 0}\right],
\end{aligned}
$$

$$
\begin{aligned}
\frac{\partial E_{30}}{\partial z}= & -\frac{\alpha_{3}}{2} E_{30}-\frac{\mathrm{i} \omega_{3}}{2 n_{3} c_{0}}\left[\chi_{\mathrm{THG}}^{(3)} E_{\mathrm{L} 0}^{3} \exp (\mathrm{i} \Delta k z)\right. \\
& \left.+n_{\mathrm{L}} n_{2, \mathrm{~L} 3}\left|E_{\mathrm{L} 0}\right|^{2} E_{30}+n_{3} n_{2,33}\left|E_{30}\right|^{2} E_{30}\right],
\end{aligned}
$$

where $\alpha_{\mathrm{L}}$ and $\alpha_{3}$ are the absorption coefficients at the fundamental frequency $\nu_{\mathrm{L}}=\omega_{\mathrm{L}} / 2 \pi=c_{0} \tilde{\nu}_{\mathrm{L}}=c_{o} / \lambda_{\mathrm{L}}$ and at the third-harmonic frequency $\nu_{3}=\omega_{3} / 2 \pi=c_{0} \tilde{\nu}_{3}=c_{0} / \lambda_{3}$, respectively. $c_{0}$ is the light velocity in vacuum. $\chi_{\mathrm{THG}}^{(3)}=$ $\chi_{x x x x}^{(3)}\left(-\omega_{3} ; \omega_{\mathrm{L}}, \omega_{\mathrm{L}}, \omega_{\mathrm{L}}\right)$ is the nonlinear-susceptibility component responsible for third-harmonic generation. $n_{\mathrm{L}}$ and $n_{3}$ are the linear (intensity independent) refractive indices at $\nu_{\mathrm{L}}$ and $\nu_{3}$, respectively. $n_{2, \mathrm{LL}}, n_{2, \mathrm{~L} 3}$, and $n_{2,33}$ are field coefficients of the nonlinear refractive index. $\Delta k=k_{3}-3 k_{\mathrm{L}}$ is the wavevector mismatch between the fundamental and third-harmonic wave.

The intensities of the fundamental laser light $I_{\mathrm{L}}$, and of the third harmonic light $I_{3}$, are obtained from the field amplitudes by $(i=\mathrm{L}, 3)$

$I_{i}=\frac{n_{i} \varepsilon_{0} c_{0}}{2}\left|E_{i 0}\right|^{2}$,

where $\varepsilon_{0}$ is the permittivity of vacuum.

The third-harmonic intensity-conversion efficiency is $\eta_{\mathrm{I}}=I_{3}(l) / I_{\mathrm{L}}(0)$, where $l$ is the sample length. The time- 
integrated third-harmonic conversion efficiency is $\eta_{\mathrm{TI}}=$ $\int_{-\infty}^{\infty} I_{3}(t, l) d t / \int_{-\infty}^{\infty} I_{\mathrm{L}}(t, 0) d t$ and the third-harmonic energyconversion efficiency is

$\eta_{\mathrm{E}}=\frac{\int_{0}^{\infty}\left[\int_{-\infty}^{\infty} I_{3}(t, r, l) d t\right] r d r}{\int_{0}^{\infty}\left[\int_{-\infty}^{\infty} I_{\mathrm{L}}(t, r, 0) d t\right] r d r}$.

The absorption coefficients $\alpha_{3}$ and $\alpha_{\mathrm{L}}$ of the solutions are approximately given by $(i=3, \mathrm{~L})$

$\alpha_{i}=\alpha_{\mathrm{S}, i}+\sigma_{i} N_{\mathrm{D}}$

where $\alpha_{\mathrm{S}}$ is the absorption coefficient of the solvent, $\sigma$ is the absorption cross section of the solute, and $N_{\mathrm{D}}=C N_{\mathrm{A}}$ is the number density of dye molecules, with $C$ the dye concentration and $N_{\mathrm{A}}$ the Avogadro number. The absorption data are derived from Fig. 1 and Fig. 7, and are collected in Table 1 . The absorption of the pump laser is negligibly small. Under phase-matching condition the absorption coefficient at the third-harmonic frequency limits the effective interaction length $l_{\mathrm{I}}$ to $l_{\mathrm{I}} \approx 3 \alpha_{3}^{-1}$ [19].

The linear refractive indices $n_{\mathrm{L}}$ and $n_{3}$ of the solutions are approximated by [19]

$n_{i}=\left(n_{\mathrm{S}, i}^{2}+\frac{L_{i}^{(1)}}{\varepsilon_{0}} N_{\mathrm{D}} \gamma_{\mathrm{D}, i}^{(1)^{\prime}}\right)^{1 / 2}$

where $L_{i}^{(1)}=\left(n_{\mathrm{S}, i}^{2}+2\right) / 3$ is the Lorentz local-field correction factor, and $\gamma_{\mathrm{D}, i}^{(1)^{\prime}}=\gamma_{\mathrm{D}, x x}^{(1)^{\prime}}\left(-\nu_{i} ; \nu_{i}\right)$ is the real part of the linear polarizability of the dye molecules. The refractive indices of the solvents TFE and HFIP and of the solutions of dye PMC in TFE have been determined recently [36] (see also [16, 37] for HFIP, and [38-40] for TFE). For the dye PMC in TFE the $\gamma_{\mathrm{D}}^{(1)^{\prime}}$ values are derived from (6), and for PMC in HFIP the same values of $\gamma_{\mathrm{D}}^{(1)^{\prime}}$ are used to determine $n_{\mathrm{L}}$ and $n_{3}$. The data are also collected in Table 1.

The linear wavevector mismatch $\Delta k$ is given by

$\Delta k=k_{3}-3 k_{\mathrm{L}}=6 \pi \tilde{\nu}_{\mathrm{L}}\left(n_{3}-n_{\mathrm{L}}\right)$.

At the phase-matching dye concentration $C_{\mathrm{PM}}$ it is $n_{3}=n_{\mathrm{L}}$ and $\Delta k=0$.

The third-harmonic nonlinear optical susceptibility $\chi_{\mathrm{THG}}^{(3)}=\chi_{x x x x}^{(3)}\left(-\omega_{3} ; \omega_{\mathrm{L}}, \omega_{\mathrm{L}}, \omega_{\mathrm{L}}\right)$ is composed of solvent $\chi_{\mathrm{THG}, \mathrm{S}}^{(3)}$, and dye contributions $\chi_{\mathrm{THG}, \mathrm{D}}^{(3)}$, i.e.,

$\chi_{\mathrm{THG}}^{(3)}=\chi_{\mathrm{THG}, \mathrm{S}}^{(3)}+\chi_{\mathrm{THG}, \mathrm{D}}^{(3)}$.

The relations between the third-harmonic nonlinear susceptibilities $\chi_{\mathrm{THG}}^{(3)}$ and the third-harmonic hyperpolarizabilities $\gamma_{\mathrm{THG}}^{(3)}=\gamma_{x x x x}^{(3)}\left(-\omega_{3} ; \omega_{\mathrm{L}}, \omega_{\mathrm{L}}, \omega_{\mathrm{L}}\right)$ are

$\chi_{\mathrm{THG}, \mathrm{S}}^{(3)}=\frac{N_{\mathrm{S}}}{\varepsilon_{0}} L_{\mathrm{THG}}^{(4)} \gamma_{\mathrm{THG}, \mathrm{S}}^{(3)}$

and

$\chi_{\mathrm{THG}, \mathrm{D}}^{(3)}=\frac{N_{\mathrm{D}}}{\varepsilon_{0}} L_{\mathrm{THG}}^{(4)} \gamma_{\mathrm{THG}, \mathrm{D}}^{(3)}$, where $L_{\mathrm{THG}}^{(4)}=L^{(4)}\left(\omega_{3} ; \omega_{\mathrm{L}}, \omega_{\mathrm{L}}, \omega_{\mathrm{L}}\right)=\left(n_{3}^{2}+2\right)\left(n_{\mathrm{L}}^{2}+2\right)^{3} / 81$ is the Lorentz local-field correction factor for the thirdharmonic interaction. $N_{\mathrm{S}}=\varrho N_{\mathrm{A}} / m_{\mathrm{M}}$ is the number density of solvent molecules (for TFE: density $\varrho=1.4106 \mathrm{~g} / \mathrm{cm}^{3}$, molar mass $m_{\mathrm{M}}=100.04 \mathrm{~g} / \mathrm{mol}$; for HFIP: $\varrho=1.62 \mathrm{~g} / \mathrm{cm}^{3}$, $\left.m_{\mathrm{M}}=168.04 \mathrm{~g} / \mathrm{mol}\right) . \chi_{\mathrm{THG}, \mathrm{S}}^{(3)}$ of HFIP has been determined previously [18]. $\chi_{\mathrm{THG}, \mathrm{S}}^{(3)}$ of TFE and $\chi_{\mathrm{THG}, \mathrm{D}}^{(3)}$ of the dye are determined in this work (see next section).

The electric field coefficients of the nonlinear refractive indices, $n_{2, \mathrm{LL}}, n_{2, \mathrm{~L} 3}$, and $n_{2,33}$, take care of the intensity dependence of the refractive indices. It is [19]

$n_{\mathrm{L}}(I)=n_{\mathrm{L}}+\frac{1}{2} n_{2, \mathrm{LL}}\left|E_{\mathrm{L} 0}\right|^{2}+\frac{1}{2} n_{2, \mathrm{~L} 3}\left|E_{30}\right|^{2}$,

and

$n_{3}(I)=n_{3}+\frac{1}{2} n_{2,33}\left|E_{30}\right|^{2}+\frac{1}{2} \frac{n_{\mathrm{L}}}{n_{3}} n_{2, \mathrm{~L} 3}\left|E_{\mathrm{L} 0}\right|^{2}$.

The field coefficients of the nonlinear refractive indices are related to the third-order nonlinear-susceptibility components by

$n_{2, i j}=\frac{D_{i j}}{n_{i}} \chi_{x x x x}^{(3)}\left(-\omega_{i} ; \omega_{j},-\omega_{j}, \omega_{i}\right)$,

( $i=\mathrm{L}, 3 ; j=\mathrm{L}, 3$ ), where $D_{i j}=3$ for $i=j$, and $D_{i j}=6$ for $i \neq j$. The electric-field coefficients of the nonlinear refractive indices have contributions from the solvent and the solute, i.e.,

$n_{2, i j}=n_{2, i j, \mathrm{~S}}+n_{2, i j \mathrm{D}}$.

The solvent contributions are nearly frequency independent since the transition frequencies involved in the susceptibility expression $[20,41]$ are much larger than the laser and the third-harmonic frequency. Therefore the $n_{2, i j, \mathrm{~s}}$ values are approximately given by $n_{2,33, \mathrm{~S}} \approx n_{2, \mathrm{LL}, \mathrm{S}}=n_{2, \mathrm{~S}}$ and $n_{2, \mathrm{~L} 3, \mathrm{~S}} \approx 2 n_{2, \mathrm{~s}}$.

The $S_{0}-S_{1}$ transition frequency $\nu_{01}$ of the dye PMC is between the second- and third-harmonic frequency of the pump laser. The frequency dependence of the dye contributions to the field coefficients of the nonlinear refractive indices has to be taken into account. It is [19]

$$
\begin{aligned}
n_{2, i j \mathrm{D}}= & \frac{D_{i j}}{n_{i}} \chi_{x x x x, \mathrm{D}}^{(3)^{\prime}}\left(-\omega_{i} ; \omega_{j},-\omega_{j}, \omega_{i}\right) \\
= & \frac{D_{i j}}{n_{i}} \frac{L^{(4)}\left(\omega_{i}, \omega_{j}, \omega_{j}, \omega_{i}\right)}{\varepsilon_{0}} \\
& \times N_{\mathrm{D}} \gamma_{x x x x, \mathrm{D}}^{(3)^{\prime}}\left(-\omega_{i} ; \omega_{j},-\omega_{j}, \omega_{i}\right),
\end{aligned}
$$

where the Lorentz local-field correction factor is given by $L^{(4)}\left(\omega_{i} ; \omega_{j}, \omega_{j}, \omega_{i}\right)=\left(n_{i}^{2}+2\right)^{2}\left(n_{j}^{2}+2\right)^{2} / 81$. The real parts of the hyperpolarizabilities are roughly given by $[20,23,41]$

$$
\begin{aligned}
& \gamma_{x x x x, \mathrm{D}}^{(3)^{\prime}}\left(-\omega_{\mathrm{L}} ; \omega_{\mathrm{L}},-\omega_{\mathrm{L}}, \omega_{\mathrm{L}}\right) \approx \frac{\kappa}{\nu_{01}-\nu_{\mathrm{L}}}, \\
& \gamma_{x x x x, \mathrm{D}}^{(3)^{\prime}}\left(-\omega_{\mathrm{L}} ; \omega_{3},-\omega_{3}, \omega_{\mathrm{L}}\right) \approx \frac{\kappa}{\nu_{01}-\nu_{3}}, \\
& \gamma_{x x x x, \mathrm{D}}^{(3)^{\prime}}\left(-\omega_{3} ; \omega_{3},-\omega_{3}, \omega_{3}\right) \approx \frac{\kappa\left(\nu_{01}-\nu_{\mathrm{L}}\right)}{\left(\nu_{01}-\nu_{3}\right)^{2}} .
\end{aligned}
$$

In Sect. 4.3.2, $\kappa$ is fittet to the experimental third-harmonic conversion efficiencies at high pump-laser intensities and the nonlinear refractive-field coefficients are determined. 


\subsection{Determination of Third-Harmonic Nonlinear Susceptibilities}

In the following $\chi_{\mathrm{THG}}^{(3)}$ is determined from the third-harmonic energy-conversion efficiency $\eta_{\mathrm{E}}$ in the low pump-intensity region before the onset of saturation effects $\left(I_{0 \mathrm{~L}} \lesssim 1 \times\right.$ $10^{10} \mathrm{~W} / \mathrm{cm}^{2}$, see Figs. 4-6). In this low-intensity regime the nonlinear refractive-index effects are negligible. Without the $n_{2, i j}$ terms, (1) and (2) are analytically solvable and the expression for the third-harmonic energy-conversion efficiency of Gaussian input pump pulses given by $[19,42]$
4.3.1 Pump Laser Reduction. In $5 \mathrm{~mm}$ samples a reduction of pump-pulse transmission occurs at peak pulse intensities above $4 \times 10^{11} \mathrm{~W} / \mathrm{cm}^{2}$ for TFE and above $6 \times 10^{11} \mathrm{~W} / \mathrm{cm}^{2}$ for HFIP. Up to these peak-intensity values the saturation of the third-harmonic conversion efficiency is not influenced by any pump-pulse reduction.

The reduction of the pump-pulse transmission shown in Fig. 7 is caused by stimulated Raman scattering. Spontaneous Raman spectra of TFE are given in $[43,44]$. An infrared-absorption spectrum of HFIP is found in [45]. We performed stimulated Raman scattering experiments with pi-

$\eta_{\mathrm{E}}=\frac{4 \pi^{2} \tilde{\nu}_{3}^{2}\left\{\exp \left(-3 \alpha_{\mathrm{L}} l\right)+\exp \left(-\alpha_{3} l\right)-2 \exp \left[-\left(\alpha_{3}+3 \alpha_{\mathrm{L}}\right) l / 2\right] \cos (\Delta k l)\right\}}{3^{3 / 2} n_{3} n_{\mathrm{L}}^{3} \varepsilon_{0}^{2} c_{0}^{2}\left[\left(\alpha_{3}-3 \alpha_{\mathrm{L}}\right)^{2} / 4+\Delta k^{2}\right]}\left|\chi_{\mathrm{THG}}^{(3)}\right|^{2} I_{0 \mathrm{~L}}^{2}$.

At the phase-matching dye concentration $C_{\mathrm{PM}},\left|\chi_{\mathrm{THG}}^{(3)}\right|$ is determined by fitting (19) to $\eta_{\mathrm{E}}\left(C_{\mathrm{PM}}, I_{0 \mathrm{~L}}=9.2 \times 10^{9} \mathrm{~W} / \mathrm{cm}^{2}\right)$ in Fig. 4. The obtained values are collected in Table 1. For HFIP the solvent third-harmonic nonlinear susceptibility $\chi_{\mathrm{THG}, \mathrm{S}}^{(3)}$ is known from previous experiments. The dye's nonlinear susceptibility $\chi_{\mathrm{THG}, \mathrm{D}}^{(3)}$ is thought to be negative because $\nu_{3}$ is above the electronic $S_{0}-S_{1}$ transition frequency [23]. Under this assumption, $\chi_{\mathrm{THG}, \mathrm{D}}^{(3)}$ at $C_{\mathrm{PM}}(8)$ and $\gamma_{\mathrm{THG}, \mathrm{D}}^{(3)}$ (10) of the dye PMC in HFIP are determined (see Table 1). Assuming the same $\chi_{\text {THG,D }}^{(3)}$ value of the PMC in TFE, the $\chi_{\text {THG }, \mathrm{S}}^{(3)}$ value of TFE is determined (see Table 1 ).

It is $\chi_{\mathrm{THG}, \mathrm{S}}^{(3)} / \chi_{\mathrm{THG}}^{(3)}\left(C_{\mathrm{PM}}\right) \approx 0.49$ for the solvent HFIP and $\chi_{\mathrm{THG}, \mathrm{S}}^{(3)} / \chi_{\mathrm{THG}}^{(3)}\left(C_{\mathrm{PM}}\right) \approx 0.39$ for the solvent TFE. Under phase-matching conditions the solvent contribution to the total third-harmonic signal is 0.24 in the case of HFIP, and 0.15 in the case of TFE.

The solid curves in Fig. $4 \mathrm{a}, \mathrm{b}$ are calculated using the parameters of Table 1. The concentration-dependent fringe pattern is due to the wavevector mismatch $\Delta k$. In the experiments the concentration-dependent fringes are not observed, because there occurs an averaging over the beam divergence and over the spectral width of the pulses.

\subsection{Limitation of Third-Harmonic Conversion Efficiency}

The straight dotted curves in Figs. 5, 6 represent theoretical third-harmonic energy-conversion efficiencies versus input peak-fundamental-laser intensity according to (19). The solid curves 1 take care of the pump-pulse depletion at high input intensities [(1) and (2) without $n_{2, i j}$ contributions]. The curves belong to phase-matched interactions. At high pumppulse intensities $\left(I_{0 \mathrm{~L}} \gtrsim 2 \times 10^{10} \mathrm{~W} / \mathrm{cm}^{2}\right)$ the experimental third-harmonic conversion efficiency of the low-intensity phase-matched solutions becomes smaller than the theoretical expectations. An experimental maximum is reached at $I_{0 \mathrm{~L}} \approx 3 \times 10^{11} \mathrm{~W} / \mathrm{cm}^{2}$, well below the theoretical dotted curves and solid curves 1 .

In the following, the influences of nonlinear pump-light transmission and of intensity-dependent refractive indices on the limitiation of third-harmonic generation are analyzed. It will be shown that the conversion efficiency is limited by the destruction of phase-matching due to the intensity dependence of the refractive indices. cosecond second-harmonic light pulses of a $\mathrm{Nd}$ : glass laser (wavelength $\lambda_{2}=527 \mathrm{~nm}$, duration $\Delta t \approx 5 \mathrm{ps}$ ).

In TFE the $2967 \mathrm{~cm}^{-1} \mathrm{CH}_{2}$ symmetric-stretch vibration showed up in stimulated Raman scattering. The threshold second-harmonic pump intensity was $I_{\text {th }} \approx 2 \times 10^{10} \mathrm{~W} / \mathrm{cm}^{2}$ for a sample length of $l=4 \mathrm{~cm}$. Using the relation $[33,46]$

$g_{\mathrm{R}} I_{\mathrm{th}} l \approx 25$,

where $g_{\mathrm{R}}$ is the Raman gain factor, we estimate $g_{\mathrm{R}}(527 \mathrm{~nm}, \mathrm{TFE}) \approx 3.11 \times 10^{-10} \mathrm{cmW}^{-1}$. With $g_{\mathrm{R}} \propto$ $\nu_{\mathrm{R}}$, where $\nu_{\mathrm{R}}$ is the Raman-Stokes frequency, we estimate $g_{\mathrm{R}}(1054 \mathrm{~nm}$, TFE $) \approx 1.3 \times 10^{-10} \mathrm{cmW}^{-1}$ and $I_{\text {th }}(1054 \mathrm{~nm}$, $4 \mathrm{~cm}) \approx 5 \times 10^{10} \mathrm{~W} / \mathrm{cm}^{2}$, in reasonable agreement with the reduction of pump-laser transmission in Fig. 7.

In a $10 \mathrm{~cm}$ long sample of HFIP stimulated emission was observed above $I_{\mathrm{th}} \approx 1.5 \times 10^{10} \mathrm{~W} / \mathrm{cm}^{2}$ for three Raman lines with spectral shifts of $\delta \tilde{\nu}_{1} \approx 295 \mathrm{~cm}^{-1}, \delta \tilde{\nu}_{2} \approx$ $735 \mathrm{~cm}^{-1}$, and $\delta \tilde{\nu}_{3} \approx 2970 \mathrm{~cm}^{-1}$. Raman gain factors of $g_{\mathrm{R}}(527 \mathrm{~nm}$, HFIP $) \approx 1.7 \times 10^{-10} \mathrm{cmW}^{-1}$ and $g_{\mathrm{R}}(1054 \mathrm{~nm}$, HFIP) $\approx 8 \times 10^{-11} \mathrm{cmW}^{-1}$ are estimated (20). A threshold intensity of $I_{\mathrm{th}}(1054 \mathrm{~nm}, 4 \mathrm{~cm}) \approx 8 \times 10^{10} \mathrm{~W} / \mathrm{cm}^{2}$ is obtained which is in reasonable agreement with the onset of pump-pulse reduction in Fig. 7.

4.3.2 Intensity-Dependent Refractive Indices. The deviation of the third-harmonic energy-conversion efficiency from the theoretical solid curves 1 in Figs. 5, 6 is due to the intensity dependence of the refractive indices. This intensity dependence destroys the phase-matching and thereby limits the efficiency.

The dependence of $\eta_{\mathrm{E}}\left(C_{\mathrm{PM}}\right)$ on $I_{0 \mathrm{~L}}$ at high pumplaser intensities is used to estimate the field coefficients of the nonlinear refractive indices by fitting the solutions of (1) and (2) to the experimental data and applying the relations of (14-18). The field coefficients $n_{2, \mathrm{LL}, \mathrm{S}}$ of the solvents have been determined separately by external selffocusing studies [47] $\left(n_{2, \mathrm{LL}, \mathrm{S}}(\mathrm{HFIP}) \approx 1 \times 10^{-22} \mathrm{~m}^{2} \mathrm{~V}^{-2}\right.$, $n_{2, \mathrm{LL}, \mathrm{S}}(\mathrm{TFE}) \approx 1.2 \times 10^{-22} \mathrm{~m}^{2} \mathrm{~V}^{-2}$ ). The curves $2-7$ in Fig. 5 (PMC in TFE) and in Fig. 6 (PMC in HFIP) are calculated for different values of $\kappa(16-18)$. The dashed curves 3 apply to $\kappa=0$, i.e. the dye contribution to the nonlinear refractive index is neglected. With rising $\kappa$, the third-harmonic conversion efficiency first rises (dashed curve 2) and reaches the nonlinear refractive-index free situation (solid curve 1) for that $\kappa$ value which gives $n_{2, \mathrm{LL}}=n_{2, \mathrm{~L} 3}$ 
[see (21)]. Beyond this optimum $\kappa$ value, the third-harmonic conversion efficiency decreases continuously with increasing $\kappa$. The best fitting $\kappa$ values are $9.6 \times 10^{-44} \mathrm{Cm}^{4} \mathrm{~V}^{-3} \mathrm{~s}^{-1}$ for PMC in TFE and $1.3 \times 10^{-43} \mathrm{Cm}^{4} \mathrm{~V}^{-3} \mathrm{~s}^{-1}$ for PMC in HFIP. The obtained hyperpolarizability and nonlinear refractive index parameters are collected in Table 1.

The dash-dotted curves $1^{\prime}$ and $2^{\prime}$ in Fig. 5 belong to 0.1109 molar PMC in TFE $\left(\Delta k=-49 \mathrm{~cm}^{-1}\right)$. Curve $1^{\prime}$ excludes the nonlinear refractive effects, while curve $2^{\prime}$ applies to the best fitting nonlinear refractive-index data of the solvent TFE and of the dye $\left(\kappa=9.6 \times 10^{-44} \mathrm{Cm}^{4} \mathrm{~V}^{-3} \mathrm{~s}^{-1}\right)$. Curve $2^{\prime}$ fits reasonably well to the experimental points. Under non-phase-matched conditions the additional intensitydependent phase-mismatch due to the intensity-dependent refractive indices becomes less important.

The wavevector mismatch $\Delta k_{n 2}$ introduced at $C_{\mathrm{PM}}$ due to the intensity-dependent refractive indices is approximately given by [see (7)]

$$
\begin{aligned}
\Delta k_{n 2} & \approx 6 \pi \tilde{\nu}_{\mathrm{L}}\left(\frac{n_{2, \mathrm{~L} 3}}{2}-\frac{n_{2, \mathrm{LL}}}{2}\right)\left|E_{0 \mathrm{~L}}\right|^{2} \\
& =\frac{6 \pi \tilde{\nu}_{\mathrm{L}}}{n_{\mathrm{L}} c_{0} \varepsilon_{0}}\left(n_{2, \mathrm{~L} 3}-n_{2, \mathrm{LL}}\right) I_{\mathrm{L} 0}
\end{aligned}
$$

At $I_{0 \mathrm{~L}}=2.5 \times 10^{11} \mathrm{~W} / \mathrm{cm}^{2} \Delta k_{n 2}$ values of $-40 \mathrm{~cm}^{-1}$ for PMC in TFE and $-47 \mathrm{~cm}^{-1}$ for PMC in HFIP are estimated.

The nonlinear refractive-index-dependent phase-mismatch $\Delta k_{n 2}$ may be compensated partially by changing the dye concentration away from $C_{\mathrm{PM}}$. This behavior is seen by the dahed curve in Fig. 4b. The curve is calculated with the nonlinear refractive-index data of Table 1 for PMC in TFE at $I_{0 \mathrm{~L}}=2.5 \times 10^{11} \mathrm{~W} / \mathrm{cm}^{2}$. The optimum concentration $C_{\text {opt }}$ is shifted to a value lower than the low-intensity phase-matching concentration because of $n_{2, \mathrm{~L} 3}<n_{2, \mathrm{LL}}$. The theoretical curve agrees reasonable well with the experimental points.

The nonlinear refractive index $n_{2, \mathrm{LL}}$ causes a spectral broadening of the pump-laser pulses by self-phase modulation $[19,20]$ which is not included in (1) and (2). Differences in the refractive-index dispersion $\partial n_{\mathrm{L}} / \partial \nu$ and $\partial n_{3} / \partial \nu$ hinder a perfect phase-matching, $\Delta k=0$ (7), over the spectral width of the pulses. This dispersion over the spectral width of the pulses may reduce the third-harmonic conversion efficiency at $C_{\mathrm{opt}}$. In the following its influence is roughly estimated.

The spectral broadening of Gaussian pulses due to selfphase modulation is [48]

$\Delta \tilde{\nu}_{\mathrm{SPM}} \approx \frac{(2 \ln 2)^{1 / 2} 4 \exp (-1 / 2) n_{2, \mathrm{LL}} I_{0 \mathrm{~L}} l_{\mathrm{eff}}}{n_{\mathrm{L}} c_{0}^{2} \varepsilon_{0} \Delta t_{\mathrm{L}}}$,

where the effective interaction length $l_{\text {eff }}$ is approximately given by $l_{\text {eff }} \approx \alpha_{3}^{-1}$. The contribution of the spectral broadening $\Delta \tilde{\nu}_{\mathrm{SPM}}$ to the linear wavevector mismatch is

$\Delta k_{\mathrm{SPM}} \approx 6 \pi \tilde{\nu}_{\mathrm{L}}\left(\frac{\partial n_{3}}{\partial \tilde{\nu}}-\frac{\partial n_{\mathrm{L}}}{\partial \tilde{\nu}}\right) \Delta \tilde{\nu}_{\mathrm{SPM}}$

The reduction of the third-harmonic conversion efficiency by $\Delta k_{\text {SPM }}$ may be taken into account by replacing the sample length $l$ by an interaction length

$l^{\prime}=\min \left(l, l_{\mathrm{I}}, 3 / \Delta k_{\mathrm{SPM}}\right)$.
For PMC in TFE at $I_{0 \mathrm{~L}}=2.5 \times 10^{11} \mathrm{~W} / \mathrm{cm}^{2}$ it is $\Delta \tilde{\nu}_{\mathrm{SPM}} \approx$ $5.6 \mathrm{~cm}^{-1}$ and $\Delta k_{\mathrm{SPM}} \approx 1.8 \mathrm{~cm}^{-1}\left(\partial n_{3} / \tilde{\nu} \approx 2.8 \times 10^{-6} \mathrm{~cm}\right.$ and $\partial n_{\mathrm{L}} / \tilde{\nu} \approx 9.8 \times 10^{-7} \mathrm{~cm} \mathrm{[36])} \mathrm{resulting} \mathrm{in} l^{\prime}=l$ or $l_{\mathrm{I}}$ (see Table 1). This result shows that the influence of self-phase modulation on the third-harmonic conversion efficiency is negligible.

\section{Conclusions}

An energy-conversion efficiency of up to $1 \%$ has been achieved in frequency tripling of mode-locked Nd:glasslaser pulses in a new cyanine dye. The very low absorption coefficient of the dye at the third-harmonic frequency leads to an efficient interaction over long dye samples $\left(l_{\mathrm{I}} \approx 5 \mathrm{~mm}\right)$. Two-photon absorption and two-photon-induced excitedstate absorption are avoided since the $S_{0}-S_{1}$ transition frequency of the new dye is in the region between the second- and third-harmonic frequency. But the absence of a two-photon resonance lowers the third-order nonlinear susceptibility responsible for third-harmonic generation. The intensity-dependent refractive-index changes destroy phasematching at high pump-pulse intensities and limit thirdharmonic conversion efficiencies.

Acknowledgements. Financial support by the Deutsche Forschungsgemeinschaft and the Fonds der Chemischen Industrie is gratefully acknowledged. The authors thank the Rechenzentrum of the University Regensburg for allocation of computer time.

\section{References}

1. F. Zernike, J.E. Midwinter: Applied Nonlinear Optics (Wiley, New York 1973)

2. J.F. Reintjes: In Laser Handbook, Vol. 5, ed. by M. Bass, M.L. Stitch (North-Holland, Amsterdam 1985) pp. 1

3. V.G. Dmitriev, G.G. Gurzadyan, D.N. Nikogosyan: Handbook of Nonlinear Optical Crystals, Springer Ser. Opt. Sci., Vol. 64 (Springer, Berlin, Heidelberg 1991)

4. A.G. Arutunyan, G.R. Buniatyan, A.A. Melkonyan, G.A. Paityan: In XIIth Conf. on Coherent and Nonlinear Optics, Abstracts, Vol. 1 (Moscow State Univ. Press, Moscow 1985) pp. 78

5. A. Penzkofer, F. Ossig, P. Qiu: Appl. Phys. B 47, 71 (1988)

6. P. Qiu, A. Penzkofer: Appl. Phys. B 45, 225 (1988)

7. D.C. Hanna, M.A. Yuratich, D. Cotter: Nonlinear Optics of Free Atoms and Molecules, Springer Ser. Opt. Sci., Vol. 17 (Springer, Berlin, Heidelberg 1979)

8. J.F. Young, G.C. Bjorklund, A.H. Kung, R.B. Miles, S.E. Harris: Phys. Rev. Lett. 27, 1551 (1971)

9. R.B. Miles, S.E. Harris: IEEE J. QE-9, 470 (1973)

10. D.M. Bloom, G.W. Bekkers, J.F. Young, S.E. Harris: Appl. Phys. Lett. 26, 687 (1975)

11. D.M. Bloom, J.F. Young, S.E. Harris: Appl. Phys. Lett. 27, 390 (1975)

12. H.P. Puell, K. Spanner, W. Falkenstein, W. Kaiser, C.R. Vidal: Phys. Rev. A 14, 2240 (1976)

13. Y. Ohashi, Y. Ishibashi, T. Kobayasi, H. Inaba: Jpn. J. Appl. Phys. 15, 1817 (1976)

14. P.P. Bey. J.F. Guiliani, H. Rabin: IEEE J. QE-7, 86 (1971)

15. R.K. Chang, L.K. Galbraith: Phys. Rev. 171, 993 (1968)

16. J.C. Diels, F.P. Schäfer: Appl. Phys. 5,197 (1974)

17. L.I. Al'Perovich, T.B. Baveav, V.V. Shabalov: Sov. J. Appl. Spectrosc. 26, 196 (1977)

18. W. Leupacher, A. Penzkofer, B. Runde, K.H. Drexhage: Appl. Phys. B 44, 133 (1987) 
19. A. Penzkofer, W. Leupacher: Opt. Quantum Electron. 20, 227 (1988)

20. Y.R. Shen: The Principles of Nonlinear Optics (Wiley, New York 1984)

21. J.F. Reintjes: Nonlinear Optical Parametric Processes in Liquids and Gases (Academic, Orlando 1984)

22. J. Zemlicka, Z. Arnold: Collect. Czech. Chem. Commun. 26, 2852 (1961)

23. W. Leupacher, A. Penzkofer: Appl. Phys. 36, 25 (1985)

24. A. Penzkofer, W. Leupacher: Opt. Quantum. Electron. 19, 327 (1987)

25. H. Schillinger, A. Penzkofer: Opt. Commun. 68, 45 (1988)

26. A., Penzkofer, F. Graf: Opt. Quantum. Electron 17, 219 (1985)

27. D. von der Linde, O. Bernecker, A. Laubereau: Opt. Commun. 2, 215 (1970)

28. A. Penzkofer, D. von der Linde, A. Laubereau: Opt. Commun. 4, 377 (1972)

29. N. Bloembergen, IEEE J. QE-10, 375 (1974)

30. D. Milam: In Laser Induced Damage in Optical Materials, 1978, ed. by A.J. Glass, A.H. Guenther, NBS Special Publication 541 (US GPO, Washington, DC 1978) p. 164

31. D.C. Brown: High-Peak-Power Nd:Glass Laser Systems, Springer Ser. Opt. Sci., Vol. 25 (Springer, Berlin, Heidelberg, 1981) pp. 170

32. W. Koechner: Solid-State Laser Engineering, 2nd edn., Springer Ser. Opt. Sci., Vol. 1 (Springer, Berlin, Heidelberg 1988) p. 540
33. A. Penzkofer, A. Laubereau, W. Kaiser: Prog. Quantum. Electron. 6, 55 (1979)

34. A. Penzkofer, W. Kaiser: Opt. Quantum. Electron. 9, 315 (1977)

35. R.R. Alfano (ed.): The Supercontinuum Laser Source (Springer, Berlin, Heidelberg 1989)

36. E. Thiel, E. Horstkotte, K.H. Drexhage: To be published.

37. Technical report DP-4A of Dupont on hexafluoroisopropanol (1968)

38. J.R. Krivacic, D.W. Urry: Anal. Chem. 42, 596 (1970)

39. F. Swarts: Comptes Rendues 197, 1261 (1933)

40. C.T. Lin, A.D.S. Marques, F.B.T. Pessine, W.O.N. Guimaraes: J. Mol. Struct. 73, 159 (1981)

41. R.W. Terhune, P.D. Maker: In Lasers, Vol. 2, ed. by A.K. Levine (Dekker, New York 1968) Chap. 4

42. M. Thalhammer, A. Penzkofer: Appl. Phys. B 32, 137 (1983)

43. J. Travert, J.C. Lavalley: Spectrochim. Acta 32A, 637 (1976)

44. V.F. Kalasinsky, H.V. Anjaria: J. Phys. Chem. 84, 1940 (1980)

45. C.J. Pouchert: The Aldrich Library of Infrared Spectra, 2nd edn. (Aldrich Chemical Company, Milwaukee 1975)

46. A. Laubereau, W. Kaiser: Rev. Mod. Phys. 50, 3607 (1978)

47. B. Meier, A. Penzkofer: Appl. Phys. B 49, 513 (1989)

48. B Meier, P. Weidner, A. Penzkofer: Appl. Phys. B 51, 404 (1990)

49. R.W. Minck, R.W. Terhune, C.C. Wang: Appl. Opt. 5, 1595 (1966) 